\title{
Comprehensive immune complexome analysis detects disease-specific immune complex antigens in seminal plasma and follicular fluids derived from infertile men and women
}

\author{
Naoko Murakami ${ }^{\mathrm{a}}$, Michio Kitajima ${ }^{\mathrm{a}, *}$, Kaname Ohyama, ${ }^{\mathrm{b}, * *}$, Nozomi Aibara $^{\mathrm{b}}$, Ken Taniguchi ${ }^{\mathrm{a}}$, \\ Mian $\mathrm{Wei}^{\mathrm{c}}$, Yuriko Kitajima ${ }^{\mathrm{a}}$, Kiyonori Miura ${ }^{\mathrm{a}}$, Hideaki Masuzaki ${ }^{\mathrm{a}}$ \\ ${ }^{a}$ Department of Obstetrics and Gynecology, Nagasaki University Graduate School of Biomedical Sciences, 1-7-1 Sakamoto-machi, Nagasaki 852-8501, Japan \\ ${ }^{\mathrm{b}}$ Department of Pharmacy Practice, Nagasaki University Graduate School of Biomedical Sciences, 1-7-1 Sakamoto-machi, Nagasaki 852-8588, Japan \\ ${ }^{\mathrm{c}}$ Jiangsu Key Laboratory of Carcinogenesis and Intervention, China Pharmaceutical University, 24 Tongjia Alley, Gulou Qu, Nanjing 210009, China
}

\section{A R T I C L E I N F O}

\section{Keywords:}

Immune complex antigen

Immune complexome analysis

Infertility

Spermatogenic dysfunction

Endometriosis

\begin{abstract}
A B S T R A C T
Background: Autoimmune reactions and subsequent inflammation may underlie spermatogenic dysfunction and endometriosis-related infertility. The aim of this study is to identify disease-specific antigens in immune complexes (ICs) in seminal plasma (SP) and in follicular fluid (FF).

Methods: Immune complexome analysis, in which nano-liquid chromatography-tandem mass spectrometry is employed to comprehensively identify antigens incorporated into ICs in biological fluids, was performed for specimens collected from infertile couples undergoing assisted reproduction. Forty-two male patients consisting of subjects with oligozoospermia $(n=6)$, asthenozoospermia $(n=8)$, and normal semen analysis $(n=28)$. Fifty-eight female patients consisting of subjects with ovarian endometriosis $(n=10)$ and control women without disease $(n=48)$.

Results: Four disease-specific antigens were identified in subjects with oligozoospermia, while five diseasespecific antigens were detected in subjects with asthenozoospermia, some of which are involved in sprematogenesis. Eight antigens were detected only in subjects with endometriosis.

Conclusion: Functional characteristics of disease-specific antigens were found to correspond to the pathogenesis of male and female infertility. The formation of ICs may contribute to spermatogenic dysfunction and endometriosis-related infertility via loss of function of the related proteins. Immune complexome analysis is expected to be a valuable tool for the investigation of novel diagnostic methods and treatment strategies for infertility.
\end{abstract}

\section{Introduction}

Local immunity may play an important role in human reproduction, and disorders in local immunity can be the cause of male and female infertility $[1,2]$.

Spermatogenic dysfunction is a major cause of male infertility, though its pathogenesis is not fully understood. Inflammation in the male reproductive tract may disrupt spermatogenesis and sperm function. Destruction of testicular microstructures can induce immunity against sperm [3]. Although autoimmune orchitis and epididymitis may be rare occurrences, these conditions may relate to immunological male infertility [4]. However, even subclinical inflammation and local autoimmune reaction in the male reproductive tract can be a cause of male infertility. To permit normal spermatogenesis and fertility, the

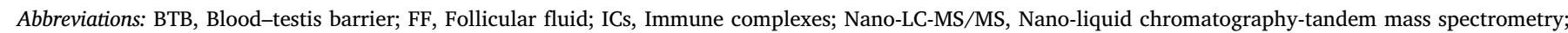
SP, Seminal plasma

* Corresponding author at: Department of Obstetrics and Gynecology, Nagasaki University Graduate School of Biomedical Sciences, 1-7-1 Sakamoto-machi, Nagasaki 852-8501, Japan.

** Corresponding author at: Department of Pharmacy Practice, Nagasaki University Graduate School of Biomedical Sciences, 1-7-1 Sakamoto-machi, Nagasaki 8528588, Japan.

E-mail addresses: cyjbt401@ybb.ne.jp (N. Murakami), m-kita@nagasaki-u.ac.jp (M. Kitajima), k-ohyama@nagasaki-u.ac.jp (K. Ohyama), bb55317401@ms.nagasaki-u.ac.jp (N. Aibara), kentaniguchi@eiseikai-mc.com (K. Taniguchi), weimian@stu.cpu.edu.cn (M. Wei), yurikokitajima@nagasaki-u.ac.jp (Y. Kitajima), kiyonori@nagasaki-u.ac.jp (K. Miura), bunbuku@nagasaki-u.ac.jp (H. Masuzaki). 
mammalian testis is maintained as an immune-privileged organ wherein immunogenic germ cells are protected from immune surveillance [1]. The Sertoli cell barrier, also known as the blood-testis barrier (BTB), plays an important role in the construction of this unique microenvironment [5]. When the BTB is impaired due to infection, injury, or obstruction of genital ducts, a large amount of sperm antigen is exposed to immune cells by leakage or infiltration, leading to sperm immunity [6]. Inflammation of the testis may affect male reproductive function. Subacute and chronic inflammation of the testis and epididymis are asymptomatic in the most patients, and there are no reliable clinical diagnostic measures [3]. As a local body fluid of the male reproductive tract, seminal plasma (SP), mediates male fertility by supporting sperm metabolism, modulating sperm function, and protecting sperm against the damage induced by the immune system via suppression of immune activity $[7,8]$. SP, which is formed by secretion from male reproductive organs including the epididymis, seminal vesicles, prostate gland, and Cowper's gland, contains many kinds of tissue-specific proteins, reflecting the afore-mentioned specific characteristics of male reproductive organs. Most of these tissue-specific proteins are intracellular proteins, including those associated with the membrane, cytoplasm, and nucleus [9-11]. The high concentration of intracellular proteins in SP may reflect the fact that most body cells interact with plasma and release components into the plasma during cell damage or death. [12]. As a result, these tissue- and cell-specific proteins are abundant in SP compared to serum or urine. Although only $10 \%$ of the protein components of SP originate from testis or epididymis, these proteins play an important role in sperm quality, are associated with testicular function, and have been implicated in male reproductive disorders [8-11,13]. Thus, changes in composition of the SP proteome may reflect a pathological process in male reproductive disorders [9].

Endometriosis is a chronic pelvic inflammatory disease and one of the major causes of female infertility. Endometriosis is characterized by the presence of ectopic endometrial tissue outside of the uterine cavity, and is manifested by chronic local inflammation in the pelvis. As with spermatogenic dysfunction, the pathogenesis of endometriosis is enigmatic. However, local immunity in the female pelvis also may be involved in the pathogenesis of endometriosis-related infertility. The mechanisms of endometriosis-related infertility vary, and include peritoneal adhesion, dysfunctional uterotubal motility, disturbed folliculogensis, and detrimental effects on spermatozoa [14,15]. Ovarian endometriotic lesions (endometriomas) are one of the main disease phenotypes, and local inflammatory reactions surrounding endometriomas may affect folliculogenesis and the process of oocyte maturation [14]. In fact, the ovary affected by endometriomas may show fibrosis and altered folliculogenesis, effects that may result from local chronic inflammation [16,17]. These findings imply that the local follicular milieu may be disturbed by endometriosis. On the other hand, several studies have reported a relationship between endometriosis and autoimmune disease $[18,19]$. In these reports, endometriosis shows symptoms similar to those of autoimmune disease. In addition, various auto-antibodies are produced in subjects with endometriosis [20]. The contents of follicular fluid (FF) are formed by osmotic pressure gradient generated by hyaluronan and versican recruits blood exudate into the follicle. Antibodies can pass through the ovarian blood-follicle barriers and diffuse into FF [21,22]. Moreover, cytokines, reactive oxygen species, and antioxidants produced by inflammation may be elevated in the FF of subjects with endometriosis [23]. Due to these facts, the FF generated by subjects with endometriosis may in turn affect the growth of endometriomas [24].

As mentioned above, autoimmune reactions and subsequent inflammation may underlie spermatogenic dysfunction and endometriosis-related infertility. However, while SP and FF are local body fluids of the gonads and reproductive tract, it is unknown what component(s) of SP and FF are recognized as auto-antigens, thereby triggering inflammation in the corresponding tissues. Immune complexes
(ICs) are formed by noncovalent interactions between foreign antigens or autoantigens and antibody molecules [25]. Enhanced formation and defective clearance of ICs occurs in autoimmune diseases [26]. In order to comprehensively identify and profile constituent antigens in ICs, we developed a proteomic strategy, designated immune complexome analysis, in which ICs are separated from whole serum and then subjected to direct tryptic digestion and nano-liquid chromatographytandem mass spectrometry [27]. We have successfully used this method to identify specific antigens in circulating ICs (CIC-antigens) in serum or cerebrospinal fluid recovered from subjects with autoimmune diseases, infectious diseases, and cancers, as well as those who are liver transplant recipients [27-31].

In the present study, we applied immune complexome analysis to SP and FF collected from infertile couples undergoing assisted reproduction. The goal of this analysis was to comprehensively identify IC-antigens, with the intent of identifying those IC-antigens specific for infertile males and for infertile women with endometriosis. Formation of ICs between the specific antigens and their corresponding autoantibodies might affect the physiological functions of the antigens, possibly leading to male and female infertility. Additionally, IC formation and deposition on tissues is known to stimulate inflammatory processes via the action of the complement system. It is hoped that the present study may lead to elucidation of the pathogenesis of male infertility and endometriosis involving immune abnormality, and to the development of new therapies to treat these fertility challenges.

\section{Materials and methods}

\subsection{Patients}

All samples were collected from infertile couples undergoing assisted reproduction technology (ART) at Nagasaki University Hospital; written consent was obtained from all participating patients. Infertility is defined as the couple who suffer from the failure to achieve a clinical pregnancy after 12 months or more of regular unprotected sexual intercourse. Before ART, male subjects received semen analysis and female subjects received the test for tubal patency by hysterosalpingography or laparoscopy, ovarian function by hormonal analysis and serial transvaginal ultrasonography, pelvic pathology, such as uterine and ovarian tumors, by ultrasonography and/or MRI. The couples with azoospermia or primary ovarian insufficiency were excluded. This study was performed according to Helsinki Declaration and was approved by the Nagasaki University Hospital Ethics Committee (Research Ethics Committee Approval No. 16020804).

\section{2. $S P$}

Forty-two semen samples were collected. After complete liquefaction, an aliquot of the ejaculate was employed for semen analysis using a Makler counting chamber (Irvine Scientific, Santa Ana, CA). Semen volume $(\mathrm{mL})$, sperm concentration $\left(10^{6} / \mathrm{mL}\right)$, and motility (\%) were recorded and classified according to the WHO guidelines [32]. Subjects were divided into three groups: the oligozoospermia group $(n=6)$, which consisted of male subjects with sperm concentrations lower than $15 \times 10^{6} / \mathrm{mL}$ without regard for sperm motility; the asthenozoospermia group $(n=8)$, which consisted of male subjects with sperm motility $<40 \%$ but with normal sperm concentrations $\left(>15 \times 10^{6} /\right.$ $\mathrm{mL})$; and the normal control group $(\mathrm{n}=28)$, which consisted of males for whom both sperm concentration and motility exceeded the lower reference limits of the WHO criteria ( $\left.>15 \times 10^{6} / \mathrm{mL},>40 \%\right)$. After semen analysis, the remaining semen was washed using Sperm Washing Medium $^{\oplus}$ (Irvine Scientific) and then was centrifuged at $2.0 \times 10^{4} \times g$ for $10 \mathrm{~min}$ to separate the pellet and supernatant. The resulting supernatant was frozen at $-40^{\circ} \mathrm{C}$; an aliquot $(10 \mu \mathrm{L})$ was used for the immune complexome analysis. In this study, SP samples were derived from not only men with definitive male factor infertility but also the 
couple with female factor infertility. In oligozoospemia group, all couple were primary infertility and there were no female partners with infertility factors. In asthenozoospermia group, six subjects were primary infertility and two subjects were secondary infertility, and there were three couples with female factor infertility, such as endometriosis $(\mathrm{n}=2)$ and ovulatory dysfunction $(\mathrm{n}=1)$. In normal control group, 24 subjects were primary infertility and four subjects were secondary infertility, and there were 15 couples with female factor infertility, such as tubal factor $(n=6)$, ovulatory dysfunction $(n=5)$, endometriosis $(n=3)$, and woman with anti-centromere antibody $(n=1)$.

\section{3. $F F$}

Fifty-eight samples were collected; the subjects were divided into two groups. The endometriosis group consisted of 10 women who had been diagnosed as having one or more endometriomas at a previous operation. At the time of surgery, cystectomy was performed in six subjects, drainage and cystic wall ablation were performed in three subjects and hemilateral cystectomy and contralateral ablation were performed in one woman with bilateral lesions. The average period from surgery to oocyte retrieval was $2.8 \pm 4.1$ years (mean \pm standard deviation). The other women lacked subjective and objective clinical symptoms of endometriosis and were designated as a control group $(n=48)$. Although all subjects received pelvic examination and transvaginal ultrasonography before ART to rule out the pelvic pathology, $15(31 \%)$ of them had been undergone pelvic surgery for indications other than endometriosis, and they were confirmed not to have the disease. At the time of transvaginal oocyte retrieval, FF was collected from the first punctured follicle. After transferring the egg to the culture medium, FF was centrifuged at $2.8 \times 10^{4} \times g$ for $5 \mathrm{~min}$ and an aliquot $(10 \mu \mathrm{L})$ of the supernatant was used for immune complexome analysis.

\section{Experimental}

\subsection{Immune complexome analysis}

ICs in SP or FF were collected using Proceptor ${ }^{\mathrm{TM}}$-sepharose beads. An aliquot $(40 \mu \mathrm{L})$ of each bead type was incubated with $10 \mu \mathrm{L}$ of pooled human serum diluted with $90 \mu \mathrm{L}$ phosphate-buffered saline (PBS) for 30 min with gentle mixing. The beads were pelleted by 1 min of centrifugation and the supernatant was removed with a pipette. The beads were washed three times with $500 \mu \mathrm{L} \mathrm{PBS} /$ wash. Washed beads were suspended in $50 \mu \mathrm{L}$ of $10 \mu \mathrm{g} / \mathrm{mL}$ papain solution (0.04 M EDTA, $0.04 \mathrm{M} \mathrm{L}$-cysteine) and incubated at $37^{\circ} \mathrm{C}$ for $30 \mathrm{~min}$. Then, $50 \mu \mathrm{L}$ of $0.06 \mathrm{M}$ iodoacetamide dissolved in PBS was added to quench the papain digestion. Next, we added $100 \mu \mathrm{L}$ of $10 \mathrm{mM}$ dithiothreitol and further incubated the sample at $56^{\circ} \mathrm{C}$ for $45 \mathrm{~min}$. Then, $100 \mu \mathrm{L}$ of $55 \mathrm{mM}$ iodoacetamide was added, and the mixture was incubated in the dark at room temperature for another $30 \mathrm{~min}$. Trypsin in $0.05 \%$ acetic acid was added to yield a final concentration of $0.5 \mathrm{~g}$ of trypsin/L, and the mixture was incubated overnight at $37^{\circ} \mathrm{C}$. An aliquot $(12 \mu \mathrm{L})$ of $10 \%$ TFA in water was added to the mixture to quench the digestion. The beads were pelleted by 1 min of centrifugation; the resulting supernatant (approximately $400 \mu \mathrm{L}$ ) was recovered, vacuum-reduced to a volume of approximately $80 \mu \mathrm{L}$, and stored at $4{ }^{\circ} \mathrm{C}$ pending subsequent analysis by nano-LC-MS/MS. The peptide mixture $(1 \mu \mathrm{L})$ was injected into an LC-electrospray ionization (ESI)-MS/MS instrument (QExactive, Thermo Fisher Scientific, Waltham, MA, USA) was equipped with EASY-nLC ${ }^{\mathrm{TM}} 1200$ system consisting of a nano LC pump) and an autosampler was used for anlaysis. Peptides were deionized and were concentrated on pre-column (Acclaim PepMap ${ }^{\mathrm{TM}} 100,75 \mu \mathrm{m} \times 2 \mathrm{~cm}$, nano Viper, C18, $3 \mu \mathrm{m}, 100 \AA$, Thermo Fisher Scientific), and were subsequently separated on a nano-LC column (C18, $75 \mu \mathrm{m}$ i.d. $\times 125 \mathrm{~mm}, 3 \mu \mathrm{M}$ particle, $100 \AA$ pore size, Nikkyo Technos, Tokyo, Japan) and ion-sprayed into MS with a spray voltage of $1.5 \mathrm{kV}$. The separation was performed by using the mobile phase A $(0.1 \%$ formic acid) and mobile phase B ( $0.1 \%$ formic acid in $90 \%$ acetonitrile), employing a gradient elution from $5 \%$ to $33 \%$ mobile phase B in $100 \mathrm{~min}$, and $100 \%$ mobile phase B held for $10 \mathrm{~min}$. MS/MS data were extracted using Proteome Discoverer 1.3.1.339 (Thermo Fisher Scientific). Spectra were searched against sub-databases from the public nonredundant protein database of UniProt Knowledgebase (human, 2015.01.29 download) with the following search parameters: mass type, monoisotopic precursor and fragments; enzyme, trypsin (KR); enzyme limits, full enzymatic cleavage allowing up to two missed cleavages; peptide tolerance, $10 \mathrm{ppm}$; fragment ion tolerance, $0.8 \mathrm{Da}$; ion and ion series calculated, B and Y ions; static modification, C (carbamidomethylation); and differential modifications, M (oxidation), $\mathrm{N}$, and $\mathrm{Q}$ (deamidation). All the results were obtained by triplicate analyses. All the peptides and proteins found in the first, second and/or third analysis were counted in the numbers of identified peptides and proteins. The procedure has been described in detail in our previous publication [33].

\subsection{Statistical analysis}

Statistical analysis was performed using Stat Mate V software (ATMS, Tokyo, Japan); the significance level was defined as a $\mathrm{p}$ value $<.05$. An $\mathrm{F}$ test was used to test the normality of distributions. Continuous variables that exhibited skewed distributions by the F test were analyzed using a two-tailed Kruskal-Wallis $\mathrm{H}$ test with post-hoc Dunnett's test. Continuous variables that exhibited normal distributions by the $\mathrm{F}$ test were analyzed using a two-tailed one-way analysis of variance (ANOVA) with post-hoc Tukey-Kramer test. For female patients, age, serum anti-Müllerian hormone (AMH), and the number of retrieved oocytes were analyzed using a Mann-Whitney $U$ test. Fisher's exact test for parity was used for comparisons between two groups.

\section{Results}

\subsection{Clinical backgrounds of the male subjects provided seminal plasma}

Age and the results of semen analysis in the male subjects are summarized in Table 1 . There was no statistically significant difference in age or semen volume among the three groups. Consistent with the group-assignment criteria, sperm concentrations in the oligozoospermia group were significantly lower than those in the other groups ( $\mathrm{p}<0.05$ versus asthenozoospermia, $\mathrm{p}<0.001$ versus normal semen analysis). Similarly, there was a statistically significant difference in sperm motility, between the asthenozoospermia group and the normal group ( $\mathrm{p}<0.001$ ). We also detected a significant difference in sperm motility between the oligozoospermia and normal groups $(\mathrm{p}<0.01)$.

Table 1

Results of semen analysis.

\begin{tabular}{llll}
\hline & $\begin{array}{l}\text { Oligozoospermia } \\
(\mathrm{n}=6)\end{array}$ & $\begin{array}{l}\text { Asthenozoospermia } \\
(\mathrm{n}=8)\end{array}$ & $\begin{array}{l}\text { Normal } \\
(\mathrm{n}=28)\end{array}$ \\
\hline $\begin{array}{l}\text { Age (years) } \\
\text { Semen volume }(\mathrm{mL})\end{array}$ & $41 \pm 6$ & $39 \pm 3$ & $38 \pm 6$ \\
$\begin{array}{c}\text { Sperm concentration } \\
\left(10^{6} / \mathrm{mL}\right)\end{array}$ & $4 \pm 1^{\mathrm{a}, \mathrm{b}}$ & $2.8 \pm 1.6$ & $3.4 \pm 1.4$ \\
\begin{tabular}{l} 
Motility (\%) \\
\hline
\end{tabular} & $37 \pm 12^{\mathrm{a}}$ & $28 \pm 8^{\mathrm{a}}$ & $104 \pm 47$ \\
\hline
\end{tabular}

Footnote: Data are presented as mean \pm SD (standard deviation). Age, semen volume, sperm concentration, and motility were compared among the three groups. After an F test was used to check the variance among the groups, age, semen volume, and motility were analyzed using a two-tailed Kruskal-Wallis $\mathrm{H}$ test with a post-hoc Dunnett's test, while sperm concentration was analyzed using a two-tailed one-way Analysis of Variance (ANOVA) with a post-hoc Tukey-Kramer test.

${ }^{\mathrm{a}} \mathrm{p}<0.001$ versus normal semen.

${ }^{\mathrm{b}} \mathrm{p}<0.05$ versus asthenozoospermia. 
Table 2

Clinical background of female subjects who provided follicular fluid.

\begin{tabular}{|c|c|c|c|}
\hline & & $\begin{array}{l}\text { Endometriosis } \\
(\mathrm{n}=10)\end{array}$ & $\begin{array}{l}\text { Control } \\
(\mathrm{n}=48)\end{array}$ \\
\hline \multicolumn{2}{|l|}{ Age (years) } & $36 \pm 4$ & $37 \pm 4$ \\
\hline \multicolumn{2}{|c|}{ Serum AMH (ng/mL) } & $2.4 \pm 1.6$ & $3.1 \pm 2.7$ \\
\hline \multicolumn{2}{|c|}{ Number of retrieved oocytes } & $6 \pm 7$ & $8 \pm 7$ \\
\hline \multicolumn{2}{|c|}{ Nulligravida } & $7(70 \%)$ & $27(56 \%)$ \\
\hline \multicolumn{2}{|l|}{ Nullipara } & $10(100 \%)$ & $40(83 \%)$ \\
\hline \multirow[t]{2}{*}{ Stage of endometriosis } & III & $5(50 \%)$ & - \\
\hline & IV & $5(50 \%)$ & - \\
\hline \multicolumn{2}{|c|}{$\begin{array}{l}\text { Follicular fluid collected from ovary } \\
\text { with endometriomas }\end{array}$} & $6(60 \%)$ & - \\
\hline \multicolumn{2}{|l|}{ Recurrent cyst at OPU } & $5(50 \%)$ & - \\
\hline
\end{tabular}

Foot note: Age, serum AMH (anti-Müllerian hormone), and the number of retrieved oocytes are presented as mean $\pm \mathrm{SD}$ (standard deviation). To compare between groups, a Mann-Whitney $U$ test was used to analyze age, serum AMH, and the number of retrieved oocytes; a Fisher's exact test was used to analyze gravidity and parity. There were no statistically significant differences between the two groups for any of these variables.

All women in the Endometriosis group has been diagnosed histologically as having uni- or bilateral endometriotic cyst(s).

OPU, oocyte pick-up.

\subsection{Clinical backgrounds of the female subjects provided follicular fluid}

Table 2 shows the clinical backgrounds of the female subjects, including: age, serum AMH levels, the number of retrieved oocytes, the number of subjects with nulligravida, the number of subjects with nullipara, the stage of endometriosis according to the revised American Society for Reproductive Medicine system, the number of subjects from whom FF was collected from the affected side, and the number of subjects with recurrent cyst(s) at the time of oocyte retrieval [34]. There were no statistically significant differences between the two groups for any of these variables.

\subsection{Immune complexome analysis}

\subsection{1. $S P$}

Here, we present for the first time (to our knowledge) a comprehensive identification of the constituent antigens assembled into CICs in SP from male infertility patients with spermatogenesis dysfunction (oligozoospermia or asthenozoospermia), or into CICs in FF from female infertility patients with ovarian endometriosis. Among the studied subjects, we identified 391 and 327 human antigens in SP of male subjects and FF of female subjects (respectively) via immune complexome analysis.

While the majority of antigens detected in SP were observed in both SP derived from patients with spermatogenesis dysfunction (oligo- and astheno-zoospermia) and SP derived from normal controls, four antigens were found only in SP derived from subjects with oligozoospermia; these antigens were not detected in SP from the other two groups (Table 3). Among the four disease-specific antigens identified in SP of oligozoospermia, sperm protein associated with the nucleus on the $\mathrm{X}$ chromosome D (also known as SPANX-D) was found in three of six oligozoospermia subjects (50\%). The other three oligozoospermia-specific antigens included zyxin, fasciculation and elongation protein zeta2 , and a probable asparagine-tRNA ligase; these three antigens were found in two of six subjects, without a specific pattern. SP from two subjects exhibited three of these oligozoospermia-specific antigens; SP from one subject retained two of these antigens; SP from one subject retained one of these antigens; and SP from two subjects did not retain any of these antigens. There was no specific pattern regarding the number of antigens present per subject, nor did the presence of various antigens appear to relate to the severity of oligozoospemia (data not shown).
Five asthenozoospermia-specific antigens (dual specificity testisspecific protein kinase 2 (also known as TESK2), probable E3 ubiquitinprotein ligase HERC1, uncharacterized protein KIAA1109, protein arginine $\mathrm{N}$-methyltransferase 7 , and ATP-binding cassette sub-family F member 1) were detected in in SP from six of eight subjects with asthenozoospermia; these antigens were not detected in SP from the other two groups (Table 4). Each antigen was detected at a frequency of $25 \%$ within subjects with asthenozoospermia without a specific pattern. SP from two subjects retained three disease-specific antigens; SP from four patients retained one specific antigen; and SP from two patients did not retain any of these specific antigens. There was no specific pattern regarding the number of antigens present per subject, nor did the presence of various antigens appear to relate to the severity of asthenozoospermia (data not shown).

\subsection{2. $F F$}

Although the majority of 327 antigens identified in FF were present both in FF from subjects with endometriosis and in FF from those without endometriosis, eight antigens were detected only in the endometriosis group; these eight antigens were not detected in FF from the control group (Table 5). These specific antigens (fibroblast growth factor receptor 1 (also known as FGFR1), probable ubiquitin carboxylterminal hydrolase FAF-Y (also known as Deubiquitinating enzyme FAF-Y), interleukin-6 receptor subunit beta (also known as gp130), sentrin-specific protease 1 , centlein, Neuralized-like protein 4, apolipoprotein B receptor, and WSC domain-containing protein 1) in FF were detected in six of ten subjects. Each antigen was detected at a frequency of $20 \%$ among subjects with endometriosis; no specific pattern was observed. FF from one subject each retained five specific antigens, four specific antigens, and three specific antigens; FF from three subjects retained one of these antigens, while those from the remaining four subjects did not harbor any of these specific antigens. There was no specific pattern regarding the number of antigens present per subject, nor did the presence of various antigens appear to relate to the severity of endometriosis (data not shown).

\section{Discussion}

In this study, we demonstrated for the first time (to our knowledge) that disease-specific ICs are formed in the local body fluids of the reproductive tracts, such as SP and FF, obtained from infertile males and females, when assessed by proteomic immune complexome analysis. ICs are formed by the binding of immunoglobulins to self and non-self antigens to promptly recognize autoantigens or prevent the spread of non-self antigens. When excessive numbers of ICs are produced, the complexes may induce inflammation and tissue damage via activation of complement. In addition, the deposition of ICs in tissues can cause fibrosis, atrophy, and dysfunction due to type-III hypersensitivity, endothelial dysfunction, and tissue remodeling [35]. Moreover, IC-associated antigens (proteins) may lose their function due to IC formation. Thus, comprehensive analysis of ICs and their antigens may facilitate the definition of the pathogenesis of disorders relevant to immune responses and inflammation.

In this study, we demonstrated for the first time (to our knowledge) that disease-specific ICs are formed in the local body fluids of the reproductive tracts, such as SP and FF, obtained from infertile males with spermatogenesis dysfunction and females with endometriosis, when assessed by proteomic immune complexome analysis. Immune response and inflammation in the local environment may be involved in the pathogenesis of both disorders. To our knowledge, most of the diseasespecific antigens identified in this study had not previously been reported to be associated with the respective disease; nonetheless, some of these antigens may correspond to the disease pathology. The specific antigens detected in SP may function as components of BTB, DNA repair machinery, or sperm nuclear envelopes. In contrast, the proteins specifically detected in FF are known to be related to inflammation. 
Table 3

Specific antigens found only in the oligozoospermia group.

\begin{tabular}{|c|c|c|c|c|c|}
\hline \multicolumn{2}{|c|}{ Accession } & Q9BXN6 & Q15942 & Q9UHY8 & Q96I59 \\
\hline \multicolumn{2}{|c|}{ Description } & $\begin{array}{l}\text { Sperm protein associated with the nucleus on the } \mathrm{X} \\
\text { chromosome D (SPANX-D) }\end{array}$ & Zyxin & $\begin{array}{l}\text { Fasciculation and elongation } \\
\text { protein zeta-2 }\end{array}$ & $\begin{array}{l}\text { Probable asparagine-tRNA ligase, } \\
\text { mitochondrial }\end{array}$ \\
\hline \multirow[t]{6}{*}{ Case } & 1 & $\bullet$ & $\bullet$ & $\bullet$ & \\
\hline & 2 & & - & - & $\bullet$ \\
\hline & 3 & - & & & - \\
\hline & 4 & - & & & \\
\hline & 5 & & & & \\
\hline & 6 & & & & \\
\hline \multicolumn{2}{|c|}{ Frequency $(\%)^{\mathrm{a}}$} & 50 & 33.3 & 33.3 & 33.3 \\
\hline
\end{tabular}

Footnote: Four specific antigens were found in four of six subjects in the oligozoospermia group.

The table shows how many antigens were detected in each patient. No specific antigens were found in cases No. 5 and No. 6 .

O: Presence of specific antigen.

a Frequency (\%) of each antigen was calculated based on the number of oligozoospermia subjects $(n=6)$.

Table 4

Specific antigens found only in the asthenozoospermia group.

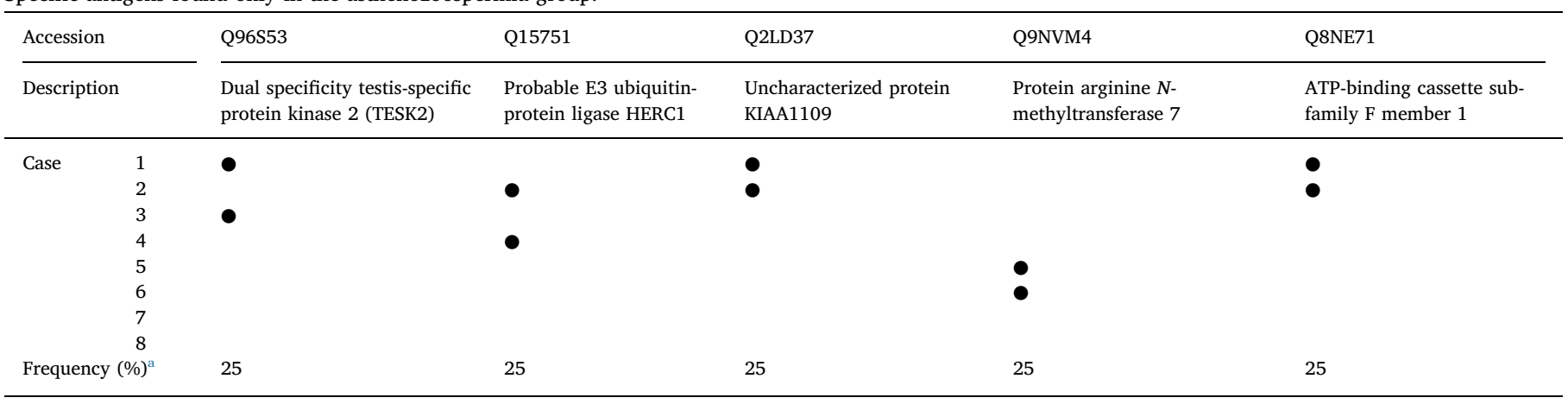

Footnote: Five specific antigens were found in six of eight subjects in the asthenozoospermia group.

The table shows how many antigens were detected in each patient. No specific antigens were found in cases No. 7 and No. 8 .

O: Presence of specific antigen.

a Frequency (\%) of each antigen was calculated based on the number of asthenozoospermia subjects $(n=8)$.

Table 5

Specific antigens found only in patients with endometriosis.

\begin{tabular}{|c|c|c|c|c|c|c|c|c|c|}
\hline \multicolumn{2}{|c|}{ Accession } & P11362 & O00507 & P40189 & Q9P0U3 & Q9NXG0 & Q96JN8 & Q0VD83 & Q658N2 \\
\hline \multicolumn{2}{|c|}{ Description } & $\begin{array}{l}\text { Fibroblast } \\
\text { growth factor } \\
\text { receptor } 1 \\
\text { (FGFR1) }\end{array}$ & $\begin{array}{l}\text { Probable ubiquitin carboxyl- } \\
\text { terminal hydrolase FAF-Y } \\
\text { (Deubiquitinating enzyme } \\
\text { FAF-Y) }\end{array}$ & $\begin{array}{l}\text { Interleukin-6 } \\
\text { receptor subunit } \\
\text { beta (gp130) }\end{array}$ & $\begin{array}{l}\text { Sentrin- } \\
\text { specific } \\
\text { protease } 1\end{array}$ & Centlein & $\begin{array}{l}\text { Neuralized- } \\
\text { like protein } 4\end{array}$ & $\begin{array}{l}\text { Apolipoprotein B } \\
\text { receptor }\end{array}$ & $\begin{array}{l}\text { WSC domain- } \\
\text { containing protein } \\
1\end{array}$ \\
\hline Case & $\begin{array}{l}1 \\
2 \\
3 \\
4 \\
5 \\
6 \\
7 \\
8 \\
9 \\
10\end{array}$ & - & • & $\bullet$ & ? & $\stackrel{\bullet}{\bullet}$ & $\bullet$ & - & • \\
\hline Frequ & $(\%)^{\mathrm{a}}$ & 20 & 20 & 20 & 20 & 20 & 20 & 20 & 20 \\
\hline
\end{tabular}

Footnote: Eight specific antigens were found in six of ten subjects in the endometriosis group.

The table shows how many antigens were detected in each patient. No specific antigens were found in cases No. 7 to No. 10.

๑: Presence of specific antigen.

${ }^{a}$ Frequency (\%) of each antigen was calculated based on the number of subjects with endometriosis $(\mathrm{n}=10)$.

Interestingly, none of the detected IC-antigens were shared among the oligozoospermia, asthenozoospermia, and endometriosis groups. These results may reflect the specific role of ICs in each disorder, implying that the analysis of ICs may contribute to expanding our knowledge of specific diseases.

Several papers have suggested an association between spermatogenic dysfunction and autoimmune response against sperm $[1,3,5,6]$. For instance, the BTB is formed by Sertoli cells in the seminiferous epithelium and plays an important role in maintaining a microenvironment (notably, an immunoprivileged compartment) that is suitable for spermatogenesis [1]. However, the BTB can be disrupted by inflammation; therefore, identification of specific BTB autoantigens 
may be crucial for understanding the pathological processes of BTB disruption [36]. In this context, among the nine disease-specific antigens identified in the SP of the oligozoospermia and asthenozoospermia groups, zyxin and TESK2 are known components of the BTB $[37,38]$. Production of antibodies against these proteins, as suggested by the presence of corresponding ICs in the present study, may lead to antigenspecific inflammation and BTB disruption.

SPANX-D, which was detected in half of SP specimens derived from oligozoospermia subjects, and the probable E3 ubiquitin-protein ligase HERC1 (HECT-type E3 ubiquitin transferase HERC1), which was specifically detected in the asthenozoospermia group, are proteins involved in DNA damage repair [39-41]. IC formation may result in disorganized sperm production due to accumulation of DNA damage and genome instability by loss of the functions of these proteins.

Among the eight specific antigens found in FF of subjects with endometriosis, several proteins might be involved in the pathogenesis of endometriosis, such as gp130, Deubiquitinating enzyme FAF-Y, and FGFR1. These proteins are known to regulate local inflammation, inflammasome formation, or the epithelial-to-mesenchymal transition (EMT) [42-45]. FF may be involved in the growth and maintenance of superficial ovarian endometriomas and peritoneal lesions [24]. Loss of function of specific antigens (which would result from excess formation of ICs) may render the local pelvic micro-environment favorable to the progression of endometriosis. IL-6 is a pro-inflammatory cytokine and may be involved in endometriosis-associated infertility [46]. The formation of ICs that include the IL-6R subunit beta (also known as gp130), which inhibits the pro-inflammatory trans-signaling cascade of IL- 6 by binding to the complex formed by IL- 6 and sIL-6R [42,47], might lead to activation of trans-signaling and exacerbation of inflammation.

On the other hand, altered folliculogenesis caused by destruction of normal ovarian cortical structures may be one of the causes of endometriosis-related infertility. Fibrotic changes in the ovarian cortex are associated with decreased follicular density and enhanced follicular recruitment and atresia $[17,18]$. Dysregulation of the inflammasome and EMT caused by the formation of ICs including Deubiquitinating enzyme FAF-Y and FGFR1, respectively, may be associated with exacerbation of inflammation and fibrosis $[48,49]$. Deubiquitinating enzyme FAF-Y may function as a polyubiquitin hydrolase that counteracts the activity of TRIM33, an E3 ubiquitin-protein ligase [43]. TRIM33 is essential for activation of the NLRP3 inflammasome [44]. FGFR1 has been shown to affect myofibroblast differentiation by inhibiting signaling by TGF- $\beta 1$ and the FGF-1 ligand, events that lead to reversion of the EMT [45]. In terms of inflammation and fibrosis, IC formation would result in activation of the complement system, a process that is known to be involved in the pathogenesis of endometriosis [50].

\section{Conclusions}

We comprehensively identified the constituent antigens of ICs in SP and FF via immune complexome analysis. Among the 391 and 327 human antigens detected in SP and FF, nine and eight antigens were found to be specific to subjects with spermatogenic dysfunction (four antigens for oligozoospemia and five antigens for asthenozoospermia) and ovarian endometriosis, respectively. Several antigens and the corresponding proteins coincide with known disease characteristics and may be involved in the pathogenesis of male and female infertility. Other specific antigens that lack known functions but were detected in SP and FF may have unknown roles in infertility. Immune complexome analysis may be a useful technique for revealing disease pathogenesis and may contribute to the development of new treatment strategies for reproductive dysfunction. However, our results, which were derived from a relatively limited number of subjects in the present work, will need to be confirmed in large-scale studies. Additionally, the exact relationship between specific antigens and male and female infertility related to spermatogenesis and endometriosis will need to be examined.
We expect that further analysis of these disease-specific antigens may provide a better understanding of the pathogenesis of both conditions.

\section{Aknowledgement}

This research was supported in part by the Grants-in-Aid for Scientific Research (grant no. 18K09294 and 16K20197 to M.K. and N.M.) from the Ministry of Education, Culture, Sports, Science and Technology of Japan.

\section{Disclosure/conflict of interest}

No potential conflicts of interest were disclosed by all the authors.

\section{References}

[1] Q. Chen, T. Deng, D. Han, Testicular immunoregulation and spermatogenesis, Semin. Cell Dev. Biol. 59 (2016) 157-165, https://doi.org/10.1016/j.semcdb.2016. 01.019.

[2] T. Zhang, C. De Carolis, G.C.W. Man, C.C. Wang, The link between immunity, autoimmunity and endometriosis: a literature update, Autoimmun. Rev. 17 (2018) 945-955, https://doi.org/10.1016/j.autrev.2018.03.017.

[3] H.C. Schuppe, A. Meinhardt, J.P. Allam, M. Bergmann, W. Weidner, G. Haidl, Chronic orchitis: a neglected cause of male infertility? Andrologia 40 (2008) 84-91, https://doi.org/10.1111/j.1439-0272.2008.00837.x.

[4] M. Fijak, A. Pilatz, M.P. Hedger, N. Nicolas, S. Bhushan, V. Michel, et al., Infectious, inflammatory and 'autoimmune' male factor infertility: how do rodent models inform clinical practice? Hum. Reprod. Update 24 (2018) 416-441, https://doi.org/ 10.1093/humupd/dmy009.

[5] G. Kaur, S. Vadala, J.M. Dufour, An overview of a Sertoli cell transplantation model to study testis morphogenesis and the role of the Sertoli cells in immune privilege, Environ. Epigenet. 3 (2017) dvx012, https://doi.org/10.1093/eep/dvx012.

[6] M.P. Hedger, Infection, immune homeostasis and immune privilege, Birkhäuser advances in infectious diseases, in: Stein-Streilein (Ed.), Immune Privilege of the Testis: Meaning, Mechanisms, and Manifestations, Springer, Basel, 2012, pp. 31-52, , https://doi.org/10.1007/978-3-0348-0445-5_2.

[7] A. Brazdova, H. Senechal, G. Peltre, P. Poncet, Immune aspects of female infertility, Int. J. Fertil. Steril. 10 (2016) 1-10.

[8] M. Camargo, P. Intasqui, R.P. Bertolla, Understanding the seminal plasma proteome and its role in male fertility, Basic Clin. Androl. 28 (2018) 6, , https://doi.org/10. 1186/s12610-018-0071-5.

[9] A.P. Drabovich, P. Saraon, K. Jarvi, E.P. Diamandis, Seminal plasma as a diagnostic fluid for male reproductive system disorders, Nat. Rev. Urol. 11 (2014) 278-288, https://doi.org/10.1038/nrurol.2014.74.

[10] J.M. Bieniek, A.P. Drabovich, K.C. Lo, Seminal biomarkers for the evaluation of male infertility, Asian J. Androl. 18 (2016) 426-433, https://doi.org/10.4103/ 1008-682X.175781.

[11] I. Batruch, I. Lecker, D. Kagedan, C.R. Smith, B.J. Mullen, E. Grober, et al., Proteomic analysis of seminal plasma from normal volunteers and post-vasectomy patients identifies over 2000 proteins and candidate biomarkers of the urogenital system, J. Proteome Res. 10 (2011) 941-953, https://doi.org/10.1021/pr100745u.

[12] N.L. Anderson, M. Polanski, R. Pieper, T. Gatlin, R.S. Tirumalai, T.P. Conrads, et al., The human plasma proteome: a nonredundant list developed by combination of four separate sources, Mol. Cell. Proteomics 3 (2004) 311-326.

[13] P. Intasqui, M. Camargo, M.P. Antoniassi, A.P. Cedenho, V.M. Carvalho, K.H.M. Cardozo, et al., Association between the seminal plasma proteome and sperm functional traits, Fertil. Steril. 105 (2016) 617-628, https://doi.org/10. 1016/j.fertnstert.2015.11.005.

[14] Y.H. Lin, Y.H. Chen, H.Y. Chang, H.K. Au, C.R. Tzeng, Y.H. Huang, Chronic niche inflammation in endometriosis-associated infertility: current understanding and future therapeutic strategies, Int. J. Mol. Sci. 19 (2018), https://doi.org/10.3390/ ijms19082385 pii: E2385.

[15] T. Tanbo, P. Fedorcsak, Endometriosis-associated infertility: aspects of pathophysiological mechanisms and treatment options, Acta Obstet. Gynecol. Scand. 96 (2017) 659-667, https://doi.org/10.1111/aogs.13082.

[16] M. Kitajima, S. Defrère, M.M. Dolmans, S. Colette, J. Squifflet, A. Van Langendonckt, et al., Endometriomas as a possible cause of reduced ovarian reserve in women with endometriosis, Fertil. Steril. 96 (2011) 685-691, https://doi.org/10. 1016/j.fertnstert.2011.06.064.

[17] M. Kitajima, M.M. Dolmans, O. Donnez, H. Masuzaki, M. Soares, J. Donnez, Enhanced follicular recruitment and atresia in cortex derived from ovaries with endometriomas, Fertil. Steril. 101 (2014) 1031-1037, https://doi.org/10.1016/j. fertnstert.2013.12.049.

[18] V.H. Eisenberg, M. Zolti, D. Soriano, Is there an association between autoimmunity and endometriosis? Autoimmun. Rev. 11 (2012) 806-814, https://doi.org/10. 1016/j.autrev.2012.01.005.

[19] L.D.G.C. Riccio, P. Santulli, L. Marcellin, M.S. Abrão, F. Batteux, C. Chapron, Immunology of endometriosis, Best Pract. Res. Clin. Obstet. Gynaecol. 50 (2018) 39-49, https://doi.org/10.1016/j.bpobgyn.2018.01.010.

[20] D. Caccavo, N.M. Pellegrino, I. Totaro, M.P. Vacca, L. Selvaggi, R. Depalo, Antilaminin-1 antibodies in sera and follicular fluid of women with endometriosis 
undergoing in vitro fertilization, Int. J. Immunopathol. Pharmacol. 24 (2011) 481-488, https://doi.org/10.1177/039463201102400221.

[21] K. Haller-Kikkatalo, A. Salumets, R. Uibo, Review on autoimmune reactions in female infertility: antibodies to follicle stimulating hormone, Clin. Dev. Immunol. 2012 (2012) 762541, https://doi.org/10.1155/2012/762541.

[22] R.J. Rodgers, H.F. Irving-Rodgers, Formation of the ovarian follicular antrum and follicular fluid, Biol. Reprod. 82 (2010) 1021-1029, https://doi.org/10.1095/ biolreprod.109.082941.

[23] G. Wu, N.A. Bersinger, M.D. Mueller, M. von Wolff, Intrafollicular inflammatory cytokines but not steroid hormone concentrations are increased in naturally matured follicles of women with proven endometriosis, J. Assist. Reprod. Genet. 34 (2017) 357-364, https://doi.org/10.1007/s10815-016-0865-3.

[24] M.O. Bahtiyar, E. Seli, E. Oral, L.M. Senturk, T.G. Zreik, A. Arici, Follicular fluid of women with endometriosis stimulates the proliferation of endometrial stromal cells, Hum. Reprod. 13 (1998) 3492-3495.

[25] R. Nezlin, A quantitative approach to the determination of antigen in immune complexes, J. Immunol. Methods 237 (2000) 1-17.

[26] A.K. Chauhan, Editorial: immune complexes in disease pathology, Front. Immunol. 8 (2017) 173, , https://doi.org/10.3389/fimmu.2017.00173.

[27] K. Ohyama, Y. Ueki, A. Kawakami, N. Kishikawa, M. Tamai, M. Osaki, et al., Immune complexome analysis of serum and its application in screening for immune complex antigens in rheumatoid arthritis, Clin. Chem. 57 (2011) 905-909, https:// doi.org/10.1373/clinchem.2010.157776.

[28] N. Aibara, K. Ichinose, M. Baba, H. Nakajima, K. Satoh, R. Atarashi, et al., Proteomic approach to profiling immune complex antigens in cerebrospinal fluid samples from patients with central nervous system autoimmune diseases, Clin. Chim. Acta 484 (2018) 26-31, https://doi.org/10.1016/j.cca.2018.05.026.

[29] K. Ohyama, N.T. Huy, H. Yoshimi, N. Kishikawa, J.E. Nishizawa, Y. Roca, et al., Proteomic profile of circulating immune complexes in chronic Chagas disease, Parasite Immunol. 38 (2016) 609-617, https://doi.org/10.1111/pim.12341.

[30] K. Ohyama, H. Yoshimi, N. Aibara, Y. Nakamura, Y. Miyata, H. Sakai, et al., Immune Complexome analysis reveals the specific and frequent presence of immune complex antigens in lung Cancer patients: a pilot study, Int. J. Cancer 140 (2017) 370-380, https://doi.org/10.1002/ijc.30455.

[31] N. Aibara, K. Ohyama, M. Hidaka, N. Kishikawa, Y. Miyata, M. Takatsuki, et al., Immune complexome analysis of antigens in circulating immune complexes from patients with acute cellular rejection after living donor liver transplantation, Transpl. Immunol. 48 (2018) 60-64, https://doi.org/10.1016/j.trim.2018.02.011.

[32] World Health Organization, WHO Laboratory Manual for the Examination and Processing of Human Semen, 5th ed., World Health Organization, Geneva, 2010.

[33] N. Aibara, C. Kamohara, A.K. Chauhan, N. Kishikawa, Y. Miyata, M. Nakashima, et al., Selective, sensitive and comprehensive detection of immune complex antigens by immune complexome analysis with papain-digestion and elution, J. Immunol. Methods 461 (2018) 85-90, https://doi.org/10.1016/j.jim.2018.06.021.

[34] American Society for Reproductive Medicine, Revised american society for reproductive medicine classification of endometriosis: 1996, Fertil. Steril. 67 (1997) 817-821.

[35] S.E. Ritzmann, J.C. Daniels, Immune complexes: characteristics, clinical correlations, and interpretive approaches in the clinical laboratory, Clin. Chem. 28 (1982) $1259-1271$
[36] M. Itoh, Testicular Autoimmunity, A Cause of Male Infertility, Springer, Tokyo, 2017, pp. 1-232, https://doi.org/10.1007/978-4-431-54460-9.

[37] N.P. Lee, D.D. Mruk, A.M. Conway, C.Y. Cheng, Zyxin, axin, and Wiskott-Aldrich syndrome protein are adaptors that link the cadherin/catenin protein complex to the cytoskeleton at adherens junctions in the seminiferous epithelium of the rat testis, J. Androl. 25 (2004) 200-215.

[38] J. Toshima, J.Y. Toshima, K. Takeuchi, R. Mori, K. Mizuno, Cofilin phosphorylation and actin reorganization activities of testicular protein kinase 2 and its predominant expression in testicular Sertoli cells, J. Biol. Chem. 276 (2001) 31449-31458, https://doi.org/10.1074/jbc.M102988200.

[39] P.F. Oliveira, C.Y. Cheng, M.G. Alves, Emerging role for mammalian target of Rapamycin in male fertility, Trends Endocrinol. Metab. 28 (2017) 165-167, https://doi.org/10.1016/j.tem.2016.12.004.

[40] K. Shimada, I. Filipuzzi, M. Stahl, S.B. Helliwell, C. Studer, D. Hoepfner, et al., TORC2 signaling pathway guarantees genome stability in the face of DNA strand breaks, Mol. Cell 51 (2013) 829-839, https://doi.org/10.1016/j.molcel.2013.08. 019.

[41] X.M. Wang, Z. Xiang, Y. Fu, H.L. Wu, W.B. Zhu, L.Q. Fan, Comparative proteomics reveal the association between SPANX proteins and clinical outcomes of artificial insemination with donor sperm, Sci. Rep. 8 (2018) 6850, , https://doi.org/10. 1038/s41598-018-25032-4.

[42] S. Rose-John, IL-6 trans-signaling via the soluble IL-6 receptor: importance for the pro-inflammatory activities of IL-6, Int. J. Biol. Sci. 8 (2012) 1237-1247, https:// doi.org/10.7150/ijbs.4989.

[43] S. Dupont, A. Mamidi, M. Cordenonsi, M. Montagner, L. Zacchigna, M. Adorno, Et. $\mathrm{Al}, \mathrm{FAM} / \mathrm{USP} 9 \mathrm{x}$, a deubiquitinating enzyme essential for TGFbeta signaling, controls Smad4 monoubiquitination, Cell 136 (2009) 123-135, https://doi.org/10. 1016/j.cell.2008.10.051.

[44] L. Weng, H. Mitoma, C. Trichot, M. Bao, Y. Liu, Z. Zhang, et al., The E3 ubiquitin ligase TRIM33 is essential for cytosolic RNAinduced NLRP3 inflammasome activation, J. Immunol. 193 (2014) 3676-3682, https://doi.org/10.4049/jimmunol. 1401448.

[45] C. Shimbori, P.S. Bellaye, J. Xia, J. Gauldie, K. Ask, C. Ramos, et al., Fibroblast growth factor-1 attenuates TGF- $\beta 1$-induced lung fibrosis, J. Pathol. 240 (2016) 197-210, https://doi.org/10.1002/path.4768.

[46] T. Iwabe, T. Harada, N. Terakawa, Role of cytokines in endometriosis-associated infertility, Gynecol. Obstet. Investig. 53 (2002) 19-25.

[47] F. Schaper, S. Rose-John, Interleukin-6: biology, signaling and strategies of blockade, Cytokine Growth Factor Rev. 26 (2015) 475-487, https://doi.org/10. 1016/j.cytogfr.2015.07.004

[48] M.N. Patel, R.G. Carroll, S. Galván-Peña, E.L. Mills, R. Olden, M. Triantafilou, et al., Inflammasome priming in sterile inflammatory disease, Trends Mol. Med. 23 (2017) 165-180, https://doi.org/10.1016/j.molmed.2016.12.007.

[49] Y.M. Yang, W.X. Yang, Epithelial-to-mesenchymal transition in the development of endometriosis, Oncotarget 8 (2017) 41679-41689, https://doi.org/10.18632/ oncotarget.16472.

[50] S. Suryawanshi, X. Huang, E. Elishaev, R.A. Budiu, L. Zhang, S. Kim, et al., Complement pathway is frequently altered in endometriosis and endometriosisassociated ovarian cancer, Clin. Cancer Res. 20 (2014) 6163-6174, https://doi.org/ 10.1158/1078-0432. 